\title{
PAK4/NAMPT Inhibitor KPT-9274
}

National Cancer Institute

\section{Source}

National Cancer Institute. PAK4/NAMPT Inhibitor KPT-9274. NCI Thesaurus. Code C126646.

An orally bioavailable inhibitor of both the serine/threonine kinase P21-activated kinase 4 (PAK4) and the nicotinamide adenine dinucleotide (NAD)-synthesizing enzyme nicotinamide phosphoribosyltransferase (NAMPT; NAMPRT ase), with potential antineoplastic activity. Upon administration, KPT-9274 allosterically binds to, destabilizes and causes degradation of PAK4. This inhibits PAK4-mediated signaling, induces cell death in, and inhibits the proliferation of PAK4-overexpressing tumor cells. In addition, KPT-9274 binds to and inhibits the activity of NAMPT. This depletes cellular NAD and inhibits NAD-dependent enzymes, both of which are needed for rapid cell proliferation; this results in tumor cell death in NAMPT-overexpressing cancer cells. PAK4, a serine/threonine kinase and member of the PAK family of proteins upregulated in various cancer cell types, regulates cell motility, proliferation and survival. NAMPT, an enzyme that is responsible for maintaining the intracellular NAD pool, plays a key role in the regulation of cellular metabolism and has cytokine-like activities. NAMPT is often overexpressed in a variety of cancers and metabolic disorders and tumor cells rely on NAMPT activity for their NAD supply. 